\title{
Single-Walled Carbon Nanotube Growth from Highly Activated Metal Nanoparticles
}

\author{
Daisuke Takagi, ${ }^{\dagger}$ Yoshikazu Homma, ${ }^{\dagger, \S, \star}$ Hiroki Hibino, ${ }^{\ddagger}$ Satoru Suzuki, ${ }^{\ddagger}$ and Yoshihiro Kobayashi ${ }^{\ddagger}, \S$ \\ ${ }^{\dagger}$ Department of Physics, Tokyo University of Science, Shinjuku, Tokyo 162-8601, Japan \\ ${ }^{\ddagger}$ NTT Basic Research Laboratories, NTT Corporation, Atsugi, Kanagawa 243-0198, Japa \\ ${ }^{\S}$ CREST, Japan Science and Technology Agency, Kawaguchi, Saitama 332-0012, Japan
}

\section{Supporting Information}

\section{Methods}

Catalyst preparation by vacuum deposition. Metals were deposited either using electron-beam or resistive-heating evaporation sources. The deposited amount was carefully controlled to be less than one monolayer (ML), i.e., 0.05-0.5 ML. When 0.15 ML or less was deposited, a high SWCNT yield could be obtained. The effect of deposition amount on the SWCNT yield for Au is shown in Figure S1.

Catalyst preparation by wet methods. Commercial Au colloidal particles with $5 \mathrm{~nm}$-diameter (MP Chemicals, http://europe.mpbio.com) were mixed with ethanol and dispersed on the substrate using a spin-coater. The rotation rate of the spin-coater was $600 \mathrm{rpm}$ for the first $30 \mathrm{~s}$ and $4000 \mathrm{rpm}$ for the next $3 \mathrm{~min}$. Similarly, diluted water solution of $\mathrm{H}\left[\mathrm{AuCl}_{4}\right]$ was dispersed on the substrate using a spin-coater. The concentration of $\mathrm{H}\left[\mathrm{AuCl}_{4}\right]$ was $0.018 \mathrm{~mol} / \mathrm{l}$.

CVD growth. CVD growth was performed using a quartz furnace $4 \mathrm{~cm}$ in diameter. Hear, the case of $850^{\circ} \mathrm{C}$ atmospheric heating and growth is described, but the atmospheric heating and the growth temperature can be set independently. First, the substrate with a deposited film or particles was introduced into the furnace which was preheated to $850{ }^{\circ} \mathrm{C}$. The introduction and heating were done in air at atmospheric pressure. The heating duration was 1-10 min depending on the deposition amount and metals. A short atmospheric heating, 30-60 s, was enough for a small deposition amount in any metal case. Then, air was evacuated with a rotary pump down to 
$1 \times 10^{2} \mathrm{~Pa}$. The temperature was kept at $850^{\circ} \mathrm{C}$ through the process. For the CVD growth, ethanol vapor was supplied by bubbling liquid ethanol using the $\mathrm{Ar}$ or $\mathrm{Ar} / \mathrm{H}_{2}$ gas for $10-30 \mathrm{~min}$ at the pressure of $5-7 \times 10^{2} \mathrm{~Pa}$. The flow rate of the bubbling gas was about $50 \mathrm{sccm}$ (standard cubic centimeter per minite). The substrates examined for nanotube growth were $\mathrm{Si}$, thermally oxidized Si, Al-hydroxide, and sapphire. The Si surface was covered with native oxide and after heat treatment in air the Si surface was thermally oxidized. Thus, the growth on Si was basically the same as that on $\mathrm{SiO}_{2}$. However, when the deposited amount of Au was small, the SWCNT yield was low. 


\section{Additional Results}

The effect of the deposited-catalyst amount. The effect of the deposition amount was examined for $\mathrm{Au}$ on $\mathrm{SiO}_{2}$ substrate. Figure $\mathrm{S} 1$ shows the SEM images for the specimens with Au catalyst of about $0.5,0.25$, and 0.05 MLs (1 ML corresponds to the Au thickness of $\sim 0.2 \mathrm{~nm}$ ). The yield of SWCNTs increase with decreasing the Au deposition amount. Note that the thick SWCNT images are created by electron beam induced conductivity (Homma, Y. et al. Appl. Phys. Lett. 2004, 84, 1750-1752). The results also show that contamination with other metals does not contribute to the SWCNT growth.

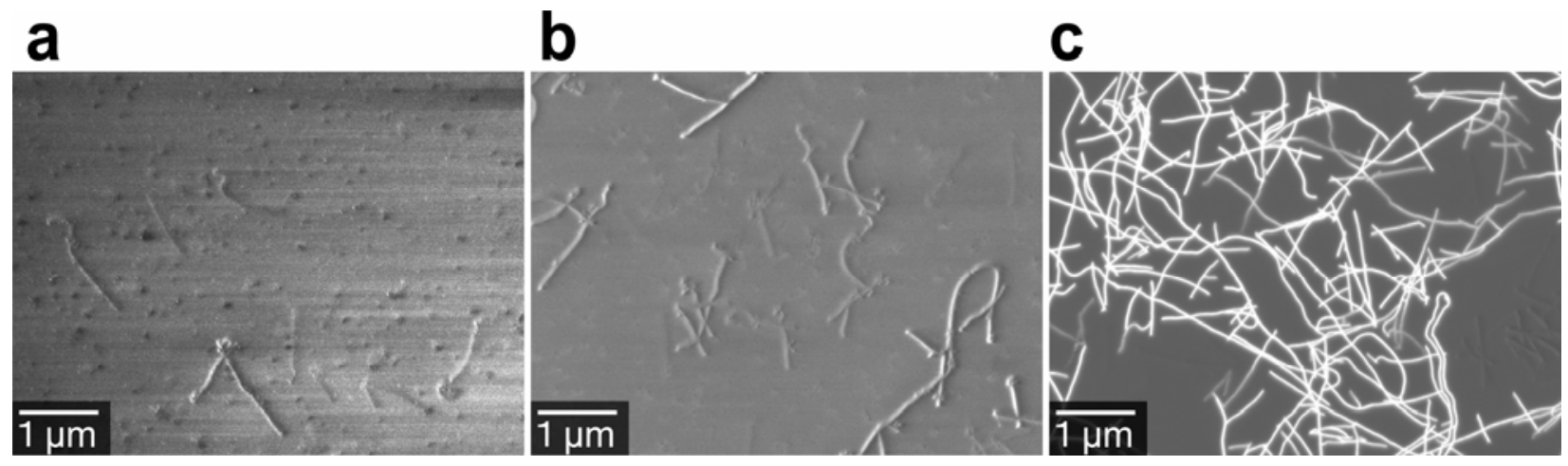

FIGURE S1. SEM images of SWCNTs grown on $\mathrm{SiO}_{2}$ substarte using Au catalyst. Au deposition amount: (a) 0.5, (b) 0.25, and (c) 0.05 MLs.

High resolution TEM image of nanotube and elemental analysis of the catalyst. A high resolution TEM image of nanotube grown from Au is shown in Figure S2(a). This clearly shows that the nanotube is single-walled. The elemental analysis of the nanocatalyst particle was not easy. We only obtained an analytical data for a 5-nm Au catalyst. The TEM image and the energy-dispersive X-ray spectrum of a $\mathrm{Au}$ catalyst particle on the $\mathrm{SiO}_{2}$ support are shown in Figures S2(b) and S2(c), respectively. The spectrum consists of peaks of $\mathrm{O}, \mathrm{Si}, \mathrm{Au}$, and $\mathrm{Cu}$. $\mathrm{Cu}$ 
peaks are caused by the $\mathrm{Cu}$ grid for specimen mounting. The spectrum indicates that the particle is gold, not Fe, Co, or Ni.

a

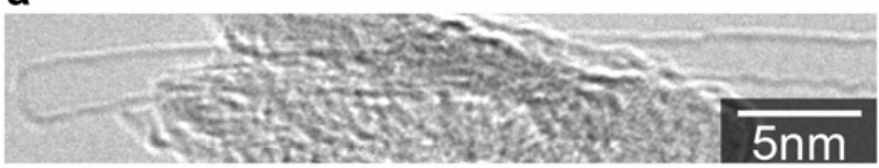

b

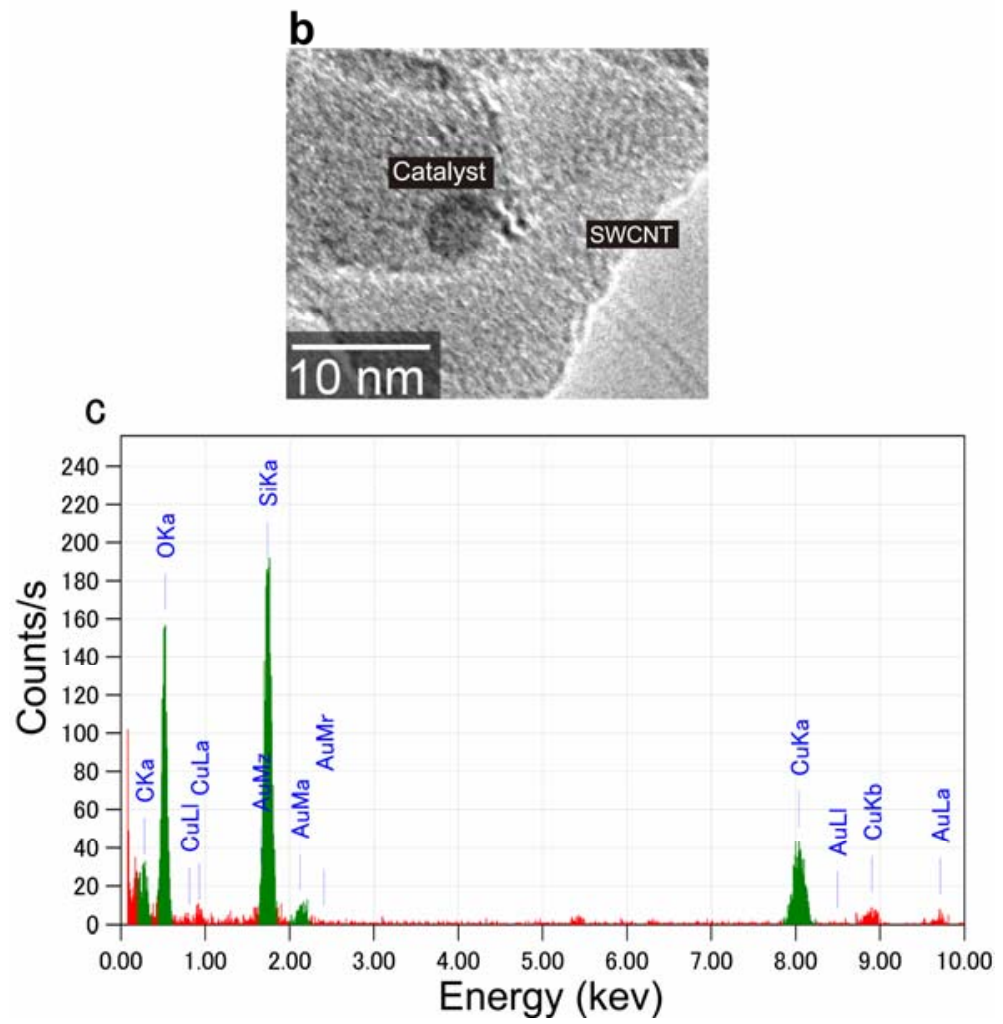

FIGURE S2. High resolution TEM image and elemental analysis of SWCNT grown from $\mathrm{Au}$ catalyst on $\mathrm{SiO}_{2}$. (a) High resolution TEM image. (b) TEM image of SWCNT from Au particle. (c) Energy-dispersive X-ray spectrum of the Au catalyst particle in (b). 